\title{
透射模式电子背散射衍射技术在纳米材料研究中的应用
}

\author{
刘紫微, 华佳捷, 林初城, 姜彩芬, 曾 毅 \\ (中国科学院 上海硅酸盐研究所, 上海 200050)
}

摘 要: 为了提高电子背散射衍射技术的空间分辨率, 使之能够对纳米尺度的晶粒或超细粉末进行相鉴定和取向分 析, 本研究通过自主设计的透射模式电子背散射衍射技术的样品台, 及调整 EBSD 探头位置及角度, 收集到清晰、 完整的透射电子菊池衍射花样。研究了减薄块体样品厚度对透射电子菊池衍射花样的影响, 对减薄氧化铅涂层样品 进行了 t-EBSD 面分布分析, 其结果的平均角度偏差(MAD)值仅为 0.38 , 成功鉴定出小于 $30 \mathrm{~nm}$ 尺度的纳米氧化铅 涂层立方相; 将超声分散后的纳米 $\mathrm{TiO}_{2}$ 粉体滴在铜网上, 采用 $\mathrm{t}-\mathrm{EBSD}$ 对纳米颗粒进行点分析, 成功鉴定了锐钠矿 相和金红石相 $\mathrm{TiO}_{2}$, 其中纳米锐钛矿 $\mathrm{TiO}_{2}$ 粉体的最小尺寸为 $20 \mathrm{~nm}$ 左右, 使得扫描电镜中相鉴定的最小分辨率从 目前的约 $100 \mathrm{~nm}$ 提高到小于 $30 \mathrm{~nm}$, 大大拓展了电子背散射衍射技术在纳米材料研究中的应用范围。

关 键 词: 透射模式电子背散射衍射; 高分辨率; 相鉴定; 纳米材料

中图分类号: TN16 文献标识码: A

\section{Application of Transmission Electron Backscattered Diffraction in Nanomaterials Research}

\author{
LIU Zi-Wei, HUA Jia-Jie, LIN Chu-Cheng, JIANG Cai-Fen, ZENG Yi
}

(Shanghai Institute of Ceramics, Chinese Academy of Sciences, Shanghai 200050, China)

\begin{abstract}
To improve the spatial resolution of electron backscatter diffraction technique, by which phase identification and orientation analysis of the nano scale grain or ultrafine powders can be achieved, a special specimen holder for transmission electron backscatter diffraction techniques (t-EBSD) accompanied by adjusting position and angle of EBSD detector, was newly designed. The new sample holder could fix the STEM samples, enabling clear TEM Kikuchi diffraction patterns to be successfully collected by EBSD detector. Then the effect of sample thickness on Kikuchi diffraction pattern was investigated. Based on the newly designed holder, the t-EBSD mapping of zirconia coating was acquired with the mean angle deviation (MAD) at only 0.38 , successfully identified the cubic phase of less than $30 \mathrm{~nm}$ zirconia coating. Put the ultrasonic dispersed $\mathrm{TiO}_{2}$ powder on copper mesh, the point analysis of t-EBSD could identify the anatase and the rutile of $\mathrm{TiO}_{2}$, distinguishing minimum size of anatase phase $\mathrm{TiO}_{2}$ less than $20 \mathrm{~nm}$. Therefore, the minimum resolution of phase identification in scanning electron microscopy is improved from about $100 \mathrm{~nm}$ to less than $30 \mathrm{~nm}$, demonstrating the electron backscatter diffraction technology a very promising way in nano materials research.
\end{abstract}

Key words: transmission electron backscattered diffraction; high resolution; phase identification; nanometer materials

收稿日期: 2015-01-29; 收到修改稿日期：2015-03-20

基金项目: 科技部国际合作专项(2013DFG52290); 中国科学院上海硅酸盐研究所科技创新重点项目(Y37ZC4141G); 上海 市无机非金属材料分析测试表征专业技术服务平台(14DZ2292900)

Fund of International Science \& Technology Cooperation Project of China (2013DFG52290); Science \& Technology Innovation Key Project of Shanghai Institute of Ceramics (Y37ZC4141G); Shanghai Technical platform for Testing and Characterization on Inorganic Materials (14DZ2292900)

作者简介: 刘紫微(1981-), 女, 硕士, 工程师. E-mail: ziweiliu@mail.sic.ac.cn

通讯作者: 曾 毅, 研究员. E-mail: zengyi@mail.sic.ac.cn 
电子背散射衍射技术 (Electron Backscattered Diffraction, EBSD)自 20 世纪 80 年代商品化以来得 到了众多材料学者关注, 成为材料研究不可或缺的 手段之一 ${ }^{[1-5]}$ 。同其他表征技术相比, EBSD 技术具 有以下特点: (1)可以在几百微米甚至毫米尺度范围 内同时给出材料显微形貌、结构、取向分布和晶粒 大小分布等多种信息; (2)对晶体取向的变化尤其敏 感, 特别适合研究材料中晶体取向的变化; (3)样品 制样简单, 可以直接分析较大的块状样品; (4)对晶 胞参数的测量精度较差, 必须要依靠能谱定量分析 ( Energy Dispersive Spectrometry, EDS)或波谱定量 分析(Wavelength Dispersive Spectroscopy, WDS)才 能进行较准确的相鉴定; (5)空间分辨率较差。电子 背散射衍射技术的相鉴定和取向分析的空间分辨率 主要取决于发生衍射的背散射电子产生范围。EBSD 分析中样品需要倾斜一定的角度, 造成电子束在水 平方向与垂直方向的分辨率略有差异。不管是水平 方向还是垂直方向的空间分辨率都远远劣于扫描电 镜(Scanning Electron Microscope, SEM)中二次电子 像或者背散射电子像的空间分辨率。

尽管 EBSD 技术在材料相鉴定的准确度和取向 分析的精确度等方面取得了很多进展, 但是其较低 的空间分辨率一直限制了 EBSD 技术的发展。EBSD 分析时采用的加速电压通常大于 $10 \mathrm{kV}$, 入射电子与 块体样品的作用范围较大, EBSD 的空间分辨率都大 于 $100 \mathrm{~nm}$ 。虽然 EBSD 方法在 SEM 分析中具有提供 晶粒取向、晶体结构以及晶粒的应变以及各类晶界 统计信息等优点 ${ }^{[6]}$, 但它在分析纳米尺度晶粒或超 细粉末时仍有很大的局限性。另外, EBSD 要求样品 被精密抛光, 在 $70^{\circ}$ 的大倾斜角度下, 表面的高度起 伏会挡住衍射电子的传输路径, 不能对不规则形状 的纳米颗粒进行分析。目前 EBSD 水平方向最佳空 间分辨率约为 $34 \mathrm{~nm}$, 垂直方向最佳约为 $38 \mathrm{~nm}^{[7]}$ 。

透射电子显微镜(Transmission Electron Microscope, TEM)的选区电子衍射(Selected Area Electron Diffraction, SAED), 是在 TEM 的投影屏上产生一组 衍射斑, 实际对应倒易点阵的某个点阵面, 通过对 衍射斑点的分析, 可确定微区的取向。由于样品较 薄或者颗粒尺寸较小, 入射电子的横向扩展区域较 小, 因此具有较高的空间分辨率。但薄样品造成倒 易点退化成倒易杆, 微小的样品转动变化不会引起 衍射斑点位置的变化, 取向的角度分辨率较低。因此, SAD 可以准确测量晶胞参数, 但对晶粒取向测量的 精度较低, 且不能做大面积的织构及取向分析。

相对于 TEM, EBSD 空间取向的精度可以达到 $0.5^{\circ}$, 但是由于电子束作用范围的制约, SEM 中的 EBSD 不能对小于 $30 \mathrm{~nm}$ 的相进行分析。因此, 近 年来不少研究人员尝试将 EBSD 与 TEM 的优势相 结合，即透射模式电子背散射衍射技术 (Transmission Electron backscattered diffraction, $\mathrm{t}-\mathrm{EBSD}$ ), 期望通过 $\mathrm{t}-\mathrm{EBSD}$ 获得具有高分辨率的 EBSD 结果。2012 年, Keller 等 ${ }^{[8]}$ 利用扫描电镜从薄 膜中获得透射电子菊池衍射花样, 使用 t-EBSD 模 式在 $40 \mathrm{~nm}$ 厚的镍薄膜上鉴定出直径 $10 \mathrm{~nm}$ 的铁钴 纳米颗粒。其研究结果还表明, 除了空间分辨率有数 量级的提高, t-EBSD 散射电子的能量宽度也小于常 规 EBSD 近两个数量级。随后, Seiichi suzuki 等 ${ }^{[9]}$ 研 $^{-}$ 究了仪器参数如倾斜角度、电镜工作距离、加速电 压和样品厚度等对 t-EBSD 表征结果的影响, 发现较 低的倾斜角度、较短的工作距离可以使空间分辨率 提高, 但是低的倾斜角度会使菊池花样的带祄度降 低; 样品厚度与加速电压对 $\mathrm{t}-\mathrm{EBSD}$ 的空间分辨率影 响较大, 不同的样品厚度对加速电压要求也不同, 需结合两者并进行适当调整才能得到较好的结果。

针对目前缺少商业化 t-EBSD 设备的现状, 本 研究自主研发了 t-EBSD 样品台, 并应用 t-EBSD 样 品台开展纳米尺度材料相分析的研究。

\section{1 实验装置}

\section{1 装置改造原理}

为了得到扫描电镜中透射电子发生布拉格衍射 后形成的菊池线, 需要将样品台进行改造。实验采 用 FEI 公司的 Magallean 400 场发射扫描电镜。图 1(a)给出了传统 EBSD 原理示意图, 图 1(b)给出了 t-EBSD 的设计原理示意图。对样品台进行改造, 磷 屏位置保持不变, 改造后的 t-EBSD 将以接收透过 样品的电子发生相应衍射后形成的菊池线为主。透 射电子菊池衍射花样产生区域的尺寸要远远小于普 通 EBSD 中来源于样品表面的衍射花样。图 2 显示 了单晶硅样品中采用蒙特卡洛模拟得到的电子扩展 范围(加速电压为 $30 \mathrm{kV}$ ), 红色代表入射电子束同单 晶硅样品作用后溢出样品表面的电子, 蓝色代表穿 透样品或被样品吸收的电子。由图 2 可知, 普通 EBSD 垂直方向的分辨率约为 300 400 nm, 而 t-EBSD 对应的水平和垂直方向的分辨率均低于 $50 \mathrm{~nm}$ 。因此, 若采用如图 1(b)所示的 t-EBSD 设计 可以得到更高的空间分辨率。

\section{$1.2 \mathrm{t}-\mathrm{EBSD}$ 装置改造问题及解决方案}

设计 $t-E B S D$ 装置需要对样品台进行重新设计, 
(a)

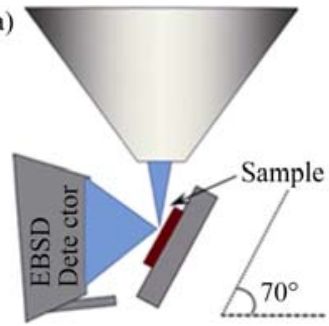

(b)

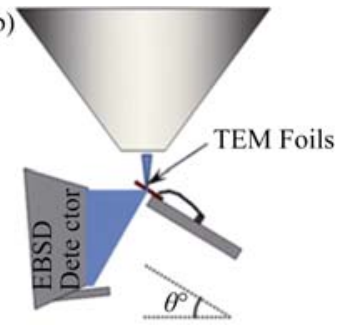

图 1 SEM 中 EBSD 与 t-EBSD 的样品放置示意图

Fig. 1 Schematic diagram of sample position laid in SEM EBSD and t-EBSD model

(a) EBSD; (b) t-EBSD

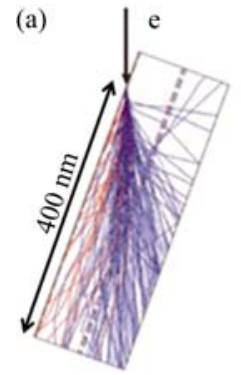

(b)

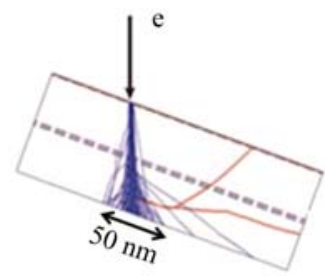

图 2 单晶硅样品中采用蒙特卡洛模拟得到的电子扩展范围 $(30 \mathrm{kV})$

Fig. 2 Monte Carlo simulations of scattering trajectories for monocrystallinesilicon (Beam energy $=30 \mathrm{kV}$ )

(a) EBSD; (b) t-EBSD

对 EBSD 探头位置及角度进行调整，使样品台可以 夹持 STEM 减薄样品, 同时 EBSD 探测器能够收集 到清晰、完整的透射电子菊池衍射花样。精准测量 物镜极靴、样品台零坐标点、EBSD 探头的收集点 位置及角度。以探头中线及物镜极靴中线的交叉点 确定 STEM 台零坐标点的水平线为准则, 使用结构 已知的(100)取向单晶硅减薄标样进行校正，对设计 加工后的样品台进行多次调整、修正，对 EDSD 的 伸缩位置及角度进行调整，确定最佳工作距离及探 头伸缩距离, 确保获得花样清晰、完整的透射电子 菊池衍射谱图。

由于 EBSD 中样品表面与投影屏表面不平行, EBSD 出厂时根据电镜厂商的探头安装位置及角度 参数内置了坐标系的矢量自动转换的设定，改装后 样品台、探测器的位置、角度发生均发生了改变, 因 此，电子束坐标系 CSbeam、样品坐标系 CSsample 及磷屏坐标系 CSscreen 的矢量变换及取向需要重新 确定。利用电子束坐标系下的矢量转变公式: 传统 EBSD 模式下样品台的倾斜角度为 $70^{\circ}$, 其旋转矢量 矩阵为 $M_{\mathrm{EBSD}}=\left(\begin{array}{ccc}0 & -\sin 70^{\circ} & \cos 70^{\circ} \\ 1 & 0 & 0 \\ 0 & \cos 70^{\circ} & \sin 70^{\circ}\end{array}\right)$; 根据上述设

计加工后的 STEM 样品的倾斜角 $-\theta$, 获得 $\mathrm{t}$-EBSD 下 的旋转矢量矩阵: $M_{\mathrm{EBSD}}=\left(\begin{array}{ccc}0 & -\sin -\theta & \cos -\theta \\ 1 & 0 & 0 \\ 0 & \cos -\theta & \sin -\theta\end{array}\right)$, t-EBSD 模式下的实际取向坐标为: 厂方软件测定 的取向坐标乘以换算系数: $\frac{M_{\mathrm{t}-\mathrm{EBSD}}}{M_{\mathrm{EBSD}}}$ 即可。

\section{2 结果与讨论}

\section{1 样品厚度对花样质量的影响}

图 3 给出了离子减薄得到的 $\mathrm{SiC} / \mathrm{BN}$ 复相陶瓷在改 造后的 t-EBSD 样品台上的 STEM 表征结果及其对应 的 t-EBSD 的菊池花样。STEM 照片中样品厚度沿着 $\mathrm{A}-\mathrm{F}$ 的顺序依次增加, $\mathrm{A}$ 点最薄, $\mathrm{F}$ 点最厚。采用 t-EBSD 对 A-F 点依次进行菊池花样的采集, 结果表明: $\mathrm{B}$ 点的菊池花样质量最佳, $\mathrm{A}$ 点几乎没有菊池花样; 从 $\mathrm{C}$ 点到 $\mathrm{F}$ 点的菊池花样逐渐变差。对于过薄的分析区 域(如 A 点), 入射电子束大部分直接穿过该区域, 发生 布拉格衍射的电子数量少, 菊池花样质量不好; 此外, 过薄区域在减薄时容易产生非晶层, 严重影响了菊池 花样的质量。随着样品厚度的增加, 能够穿透样品的电 子束减少，发生布拉格衍射的电子数量也相应减
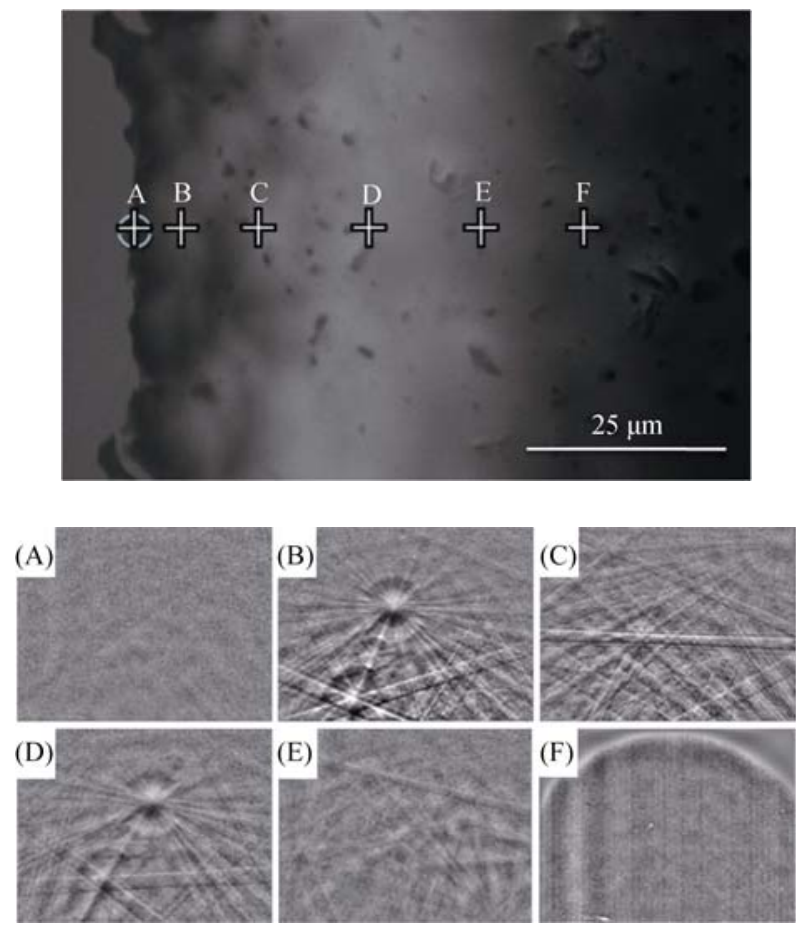

图 3 减薄 SiC/BN 复相陶瓷的 STEM 表征结果及其对应各点 的 t-EBSD 花样

Fig. 3 STEM morphology of SiC/BN compound by ion milling and the corresponding t-EBSD Kikuchi patterns 

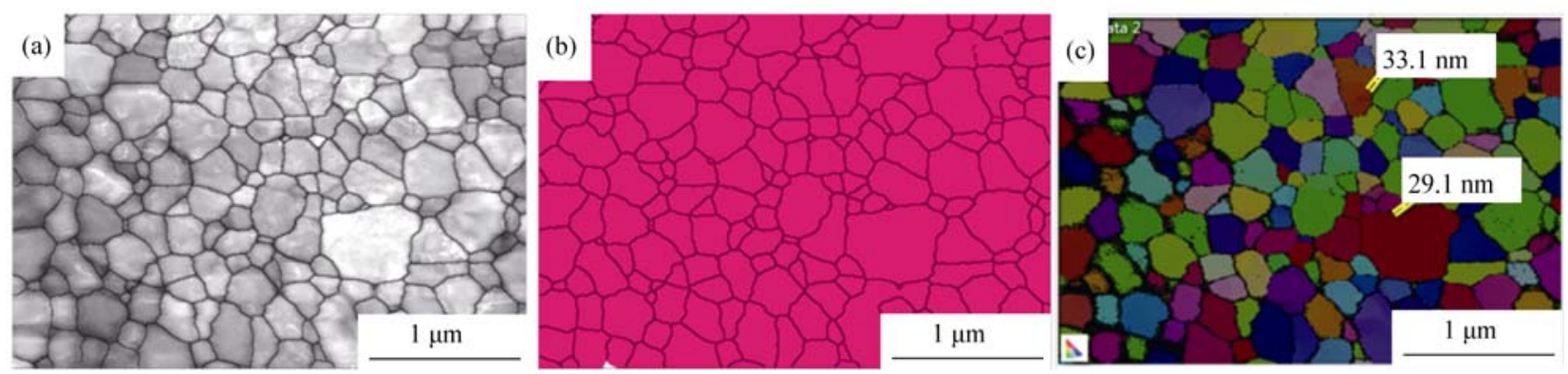

图 4 氧化锆涂层的 t-EBSD 表征结果

Fig. 4 t-EBSD characterization of zirconium oxide coating (a) Grain band contrast; (b) Phase map of zirconium oxide; c: t-EBSD of grain orientation

少, 菊池花样质量下降。因此, 要得到较好的 $\mathrm{t}-\mathrm{EBSD}$ 衍射结果, 过薄或者过厚都会影响菊池花样 质量, 必须挑选一个合适的样品厚度。

\section{2 氧化锆纳米涂层的 t-EBSD 表征}

热障氧化锆涂层广泛应用于航空发动机的叶 片等热端部件, 利用其较低的热导率降低基体承 受的热载荷, 可以起到保护基体、延长使用寿命的 作用 ${ }^{[10-11]}$ 。了解热障氧化锆涂层的晶粒相组成和大 小分布对提高其服役性能起着至关重要的作用。

用 t-EBSD 表征热障氧化锆涂层采集的菊池线 进行相鉴定与取向分布结果如图 4 所示。图 4(a)显 示了氧化锆涂层的带衬度; 图 4(b)显示该区域只有 属于立方晶系的 $\left(\left(\mathrm{ZrO}_{2}\right)_{0.88}\left(\mathrm{Y}_{2} \mathrm{O}_{3}\right)_{0.12}\right)_{0.893}$ 相存在, 其 晶胞参数为 $a=b=c=0.514 \mathrm{~nm}$; 图 4(c)为氧化锆涂层 的 t-EBSD 晶粒取向图, 该区域内的晶粒分布较不 均匀, 最大晶粒尺寸超过 $500 \mathrm{~nm}$, 同时存在一些细 小的晶粒, 可鉴别的最小晶粒仅为 $29 \mathrm{~nm}$ 。该区域 EBSD 结果的平均角度偏差(MAD)值仅为 0.38(图 5), 表明 EBSD 的结果与数据库中理论晶体学参数高度 吻合, EBSD 结果可靠。图 6(a)给出了该区域内晶粒 尺寸的分布, 图 6(b)给出了介于 $30 \sim 100 \mathrm{~nm}$ 的 $\left(\left(\mathrm{ZrO}_{2}\right)_{0.88}\left(\mathrm{Y}_{2} \mathrm{O}_{3}\right)_{0.12}\right)_{0.893}$ 相的晶粒分布, 由图可知,
采用 t-EBSD 技术可以很好地对小于 $100 \mathrm{~nm}$ 的相进 行鉴定。

\section{3 氧化钛纳米粉体的 t-EBSD 表征}

$\mathrm{TiO}_{2}$ 是一种在光催化领域获得广泛应用的材 料 ${ }^{[12-14]}$, 其常见的晶型有光催化性能较好的锐钛 矿和金红石两相。晶粒尺寸是影响 $\mathrm{TiO}_{2}$ 光催化活性 的重要因素, 晶粒越小, 晶体活性中心会更充分暴 露出来, 有利于催化活性的提高; 同时, 小晶粒的 光生载流子比大晶粒更容易通过扩散从晶粒内部迁 移到表面, 促进反应的产生, 提高光催化效率。因此, 对纳米尺度 $\mathrm{TiO}_{2}$ 颗粒的晶粒大小以及晶型进行表 征对研究其光催化性能具有重要意义。

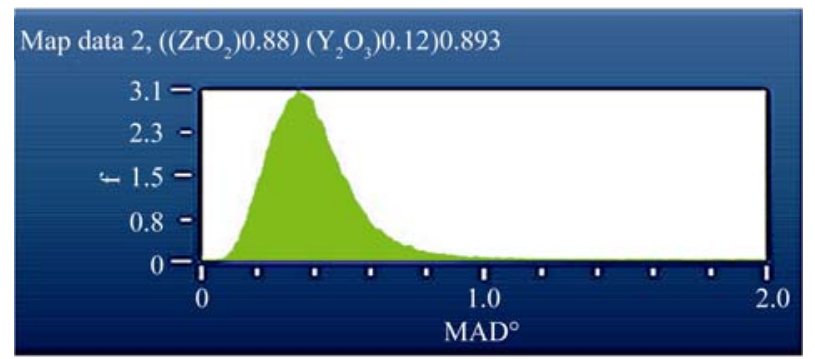

图 5 t-EBSD 菊池花样与数据库晶体结构信息匹配程度

Fig. 5 Matching degree of T-EBSD kikuchi patterns and crystalline structure information in database

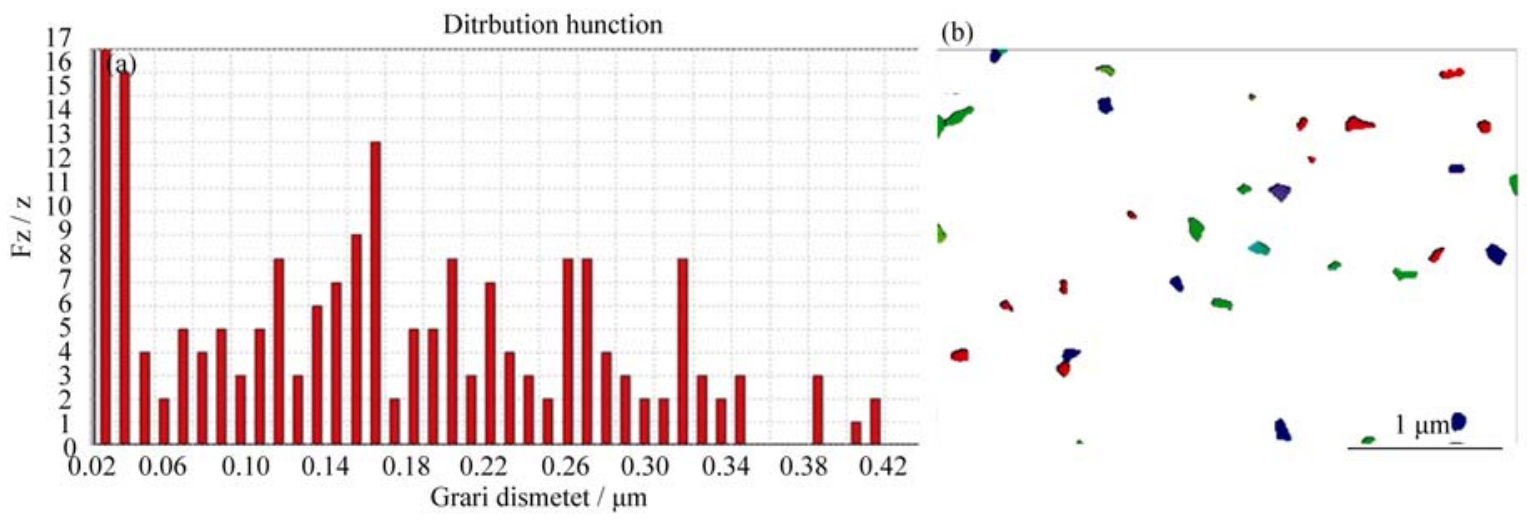

图 6 表征区域小晶粒统计

Fig. 6 Characterization of small grains

(a) Grain size distribution in the coating; (b) Distribution of zirconia crystal grains between $30-100 \mathrm{~nm}$ 
图 7 是 $\mathrm{TiO}_{2}$ 纳米粉体的二次电子像及其对应氧 化钛纳米粉体单颗粒 t-EBSD 菊池花样。二次电子 像中 $\mathrm{A}$ 标示出的一个小晶粒尺寸为 $19 \mathrm{~nm}$, 对其进 行 t-EBSD 分析, 其菊池花样如图 7(A, A')所示, 可 见, 该晶粒的菊池花样与锐钛矿相的标准菊池花样 高度吻合, 说明该相为锐钛矿的 $\mathrm{TiO}_{2}$ 。二次电子像 中 B 标示出的尺寸约为 $40 \mathrm{~nm}$ 的小晶粒进行 t-EBSD 分析, 其菊池花样见图 7(B, B') 所示, 该晶粒的菊池 花样与金红石相的标准菊池花样高度吻合, 确定这 个晶粒为金红石 $\mathrm{TiO}_{2}$ 。通过 t-EBSD 对 $\mathrm{TiO}_{2}$ 纳米粉 体的表征, 证明改造后的 t-EBSD 系统可将空间分 辨率提高到 $20 \mathrm{~nm}$ 以下。

\section{3 结论}

1) 通过精准测量物镜极靴、样品台零坐标点、 EBSD 探头的收集点位置及角度, 设计出能接受到 透射模式菊池衍射花样的 t-EBSD 装置, 应用该装 置对具有合适的厚度的减薄样品进行 t-EBSD 表征, 可获得清晰的透射电子菊池衍射图谱, 实现了对纳 米尺度晶粒的相鉴定、织构及取向差分析, 晶粒尺 寸及形状的分析, 晶界、亚晶及孪晶性质的分析等。

2) 应用 t-EBSD 装置成功地对小于 $30 \mathrm{~nm}$ 尺度的 纳米氧化锆涂层立方相进行鉴定, 相鉴定结果与数
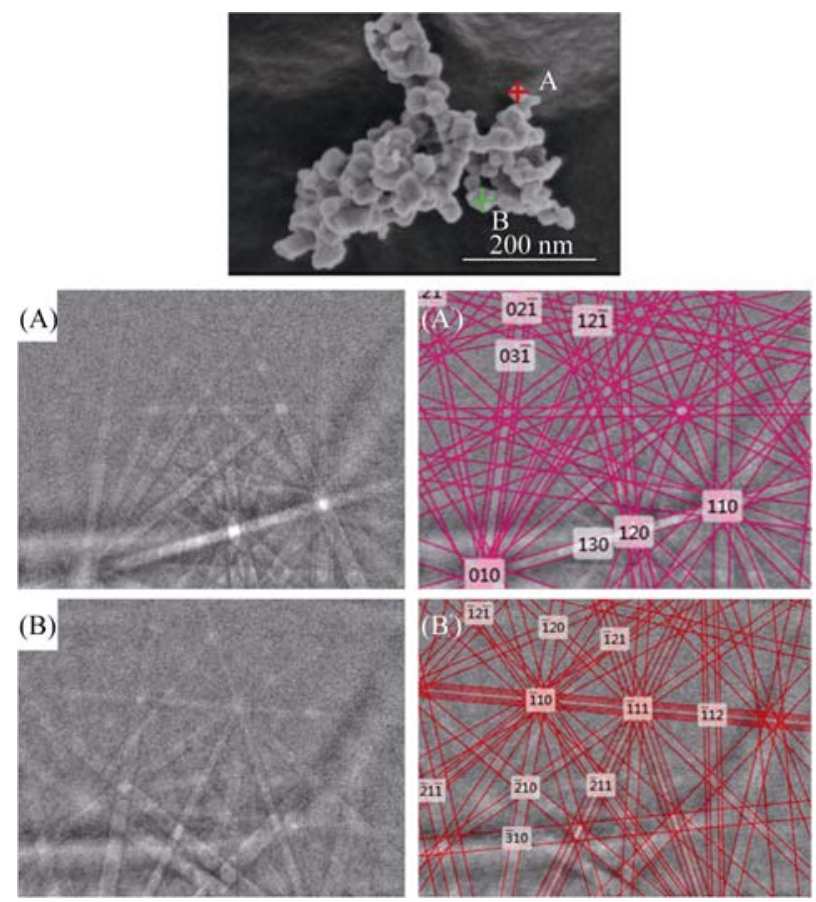

图 7 氧化钛纳米粉体的 SEM 表征结果及其对应氧化钛纳米 粉体单颗粒 t-EBSD 菊池花样

Fig. 7 SEM image of nano-titanium oxide powder and corresponding t-EBSD kikuchi patterns of nano-titanium oxide powder
据库中理论晶体学参数高度吻合, 相鉴定结果可靠。

3) t-EBSD 可以避免由于粉体表面高度起伏挡 住衍射电子的传输路径而出现的菊池花样质量严重 下降问题, 将 EBSD 的分析范围从抛光样品表面扩 展到纳米粉体样品, 成功鉴定出最小尺寸为 $20 \mathrm{~nm}$ 左右的纳米锐钛矿 $\mathrm{TiO}_{2}$ 粉体。

\section{参考文献:}

[1] KUMAR M, SCHWARTZ A J, KING W E. Microstructural evolution during grain boundary engineering of low to medium stacking fault energy fcc materials. Acta Materialia, 2002, 50(10): 2599-2612.

[2] KUMAR M, SCHWARTZ A J, KING W E. Correlating observations of deformation microstructures by TEM and automated EBSD techniques. Materials Science and Engineering, 2001, A309-310: 78-81.

[3] NISHIKAWA S, KIKUCHI S. Diffraction of cathode rays by calcite. Proceedings of the Imperial Academy, 1928, 4(8): 475-477.

[4] ALAM M N, BLACKMAN M, PASHLEY D W. High-angle kikuchi patterns. Proceedings of the royal society of London series a-Mathematical and Physical Sciences, 1954, 221(1145): 224-242.

[5] VENABLES J A, HARLAND C J. Electron backscattering patterns-new technique for obtaining crystallographic information in scanning electron-microscope. Philosophical Magazine, 1973, 27(5): 1193-1200.

[6] MINGARD K P, ROEBUCK B, BENNETT E G, et al. Comparison of EBSD and conventional methods of grain size measurement of hardmetals. International Journal of Refractory Metals and Hard Materials, 2009, 27(2): 213-223.

[7] CHEN D, KUO J C, WU W T. Effect of microscopic parameters on EBSD spatial resolution. Ultramicroscopy, 2011, 111(9/10): 1488-1494.

[8] KELLER R R, GEISS R H. Transmission EBSD from $10 \mathrm{~nm}$ domains in a scanning electron microscope. J. Microsc-Oxford, 2012, 245(3): 245-251.

[9] SUZUKI S. Evaluation of transmission-EBSD method and its application to observation of microstructures of metals. Journal of the Japan Institute of Metals, 2013, 77(7): 268-275.

[10] WANG X, ATKINSON A, CHIRIVì L, et al. Evolution of stress and morphology in thermal barrier coatings. Surface \& Coatings Technology, 2010, 204(23): 3851-3857.

[11] TRUNOVA O, BECK T, HERZOG R, et al. Damage mechanisms and lifetime behavior of plasma sprayed thermal barrier coating systems for gas turbines-Part I: Experiments. Surface \& Coatings Technology, 2008, 202(20): 5027-5032.

[12] XU S H, SHANG GUAN W F, LI D X. TiO 2 photocatalyst materials and its application in water. Environmental Science \& Technology, 2008, 31(12): 94-100.

[13] MARTA I L. Heterogeneous photocatalysis and transition metalions in photocatalytic systems. Applied Catalysis B:Environmental, 1999, 23(2/3): 89-114.

[14] VORONTSOV A V, SAVINOV E N, JIN Z S. Influence of the form of photodeposited platinum on titania upon its photocatalytic activity in CO and acetone oxidation. Journal of Photochemistry and Photobiology A: Chemistry, 1999, 125: 113-117. 